TITLE:

\title{
Direct and ultrafast probing of quantum many-body interactions through coherent two-dimensional spectroscopy: From weak- to strong-interaction regimes
}

\section{$\operatorname{AUTHOR(S):~}$}

Phuc, Nguyen Thanh; Trung, Pham Quang

\section{CITATION:}

Phuc, Nguyen Thanh ...[et al]. Direct and ultrafast probing of quantum many-body interactions through coherent two-dimensional spectroscopy: From weak- to stronginteraction regimes. Physical Review B 2021, 104(11): 115105.

ISSUE DATE:

2021-09-15

URL:

http://hdl.handle.net/2433/265263

RIGHT:

(C2021 American Physical Society 


\title{
Direct and ultrafast probing of quantum many-body interactions through coherent two-dimensional spectroscopy: From weak- to strong-interaction regimes
}

\author{
Nguyen Thanh Phuc $\odot^{*}$ \\ Department of Molecular Engineering, Graduate School of Engineering, Kyoto University, Kyoto 615-8510, Japan \\ Pham Quang Trung (i) \\ Section of Brain Function Information, Supportive Center for Brain Research, National Institute for Physiological Sciences, \\ Okazaki 444-8585, Japan
}

(Received 16 September 2020; revised 21 April 2021; accepted 25 August 2021; published 3 September 2021)

\begin{abstract}
Interactions between particles in quantum many-body systems play a crucial role in determining the electric, magnetic, optical, and thermal properties of the system. The recent progress in the laser-pulse technique has enabled the manipulations and measurements of physical properties on ultrafast timescales. Here we propose a method for the direct and ultrafast probing of quantum many-body interaction through coherent two-dimensional (2D) spectroscopy. Using a two-band fermionic Hubbard model for the minimum two-site lattice system, we find that the 2D spectrum of a noninteracting system contains only diagonal peaks; the interparticle interaction manifests itself in the emergence of off-diagonal peaks in the $2 \mathrm{D}$ spectrum before all the peaks coalesce into a single diagonal peak as the system approaches the strongly interacting limit. The evolution of the 2D spectrum as a function of the time delay between the second and third laser pulses can provide important information on the ultrafast time variation of the interaction.
\end{abstract}

DOI: 10.1103/PhysRevB.104.115105

\section{INTRODUCTION}

Important physical systems and materials often consist of a macroscopically large number of atoms, molecules, and electrons. The interaction between constituent particles can dictate various physical properties of the system, including, for example, the electrical and thermal transports and the magnetic and optical properties. However, in some metals and semiconductors, owing to the screening of the Coulomb interaction between electrons, their low-energy behaviors are similar to those of a system of noninteracting particles. These systems can be described by Landau's Fermi liquid theory [1]. In contrast, there also exist strongly correlated electronic systems beyond the Fermi liquid theory, in which the interaction between electrons cannot be ignored. Examples include the Luttinger liquid in one dimension [2,3], heavy fermionic systems [4], and systems near critical points such as the Mott-insulator phase transition [5,6]. The interparticle interaction can also result in exotic phenomena such as superfluidity and superconductivity [7]. Strong-interparticle interactions and their effects on the static and dynamic properties of the system have also been investigated extensively in ultracold atomic and molecular systems [8-12], where the ratio of the interaction energy to the kinetic energy can be varied under control. The superfluid-to-Mott-insulator phase transition was also observed in the absorption spectra of rubidium and ytterbium atoms [13,14]. However, time-resolved spectroscopies, in comparison with conventional techniques

*nthanhphuc@moleng.kyoto-u.ac.jp such as the time-of-flight and photoemission measurements, are needed for studying ultrafast phenomena in electronic and ultracold atomic systems.

Meanwhile, continual progress in the development of laser-pulse techniques has enabled a faster manipulation and measurement of the physical and chemical properties of electronic, atomic, and molecular systems [15]. This allowed us to observe intriguing nonequilibrium phenomena, such as light-induced superconductivity [16,17], ultrafast spintronics $[18,19]$, and the Floquet engineering of electronic band topology [20,21]. In this work we propose a method to investigate the effect of interaction between particles directly in quantum many-body systems on an ultrafast timescale using coherent two-dimensional (2D) spectroscopy. Coherent multidimensional, especially $2 \mathrm{D}$, spectroscopy has been widely used to study electronic excitation (exciton) and vibration dynamics in molecular and semiconductor systems [22-30]. More recently, intersubband electronic excitations in quantum wells [31], carrier dynamics in graphene [32], spin-wave [33] and fractional excitations $[34,35]$ in magnetic materials, marginal Fermi glass [36], and high-temperature superconductors [37] have been studied. In coherent 2D spectroscopy, a sequence of three laser pulses is used to excite the system, and the subsequent coherent light emission induced by the polarization of the system is measured. The $2 \mathrm{D}$ spectrum displays the emitted optical signal as a function of the frequencies $\omega_{1}$ and $\omega_{3}$, which is obtained by performing a Fourier transformation with respect to the time interval $t_{1}$ between the first two pulses and the time interval $t_{3}$ between the third pulse and the emitted signal, respectively. Physically, $\omega_{1}$ and $\omega_{3}$ amount to the excitation and emission frequencies, respectively. The 
(a)

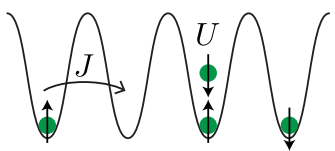

(b)

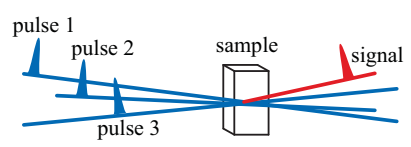

(c)

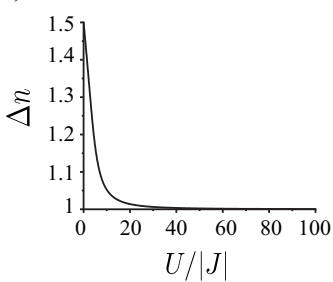

(d)

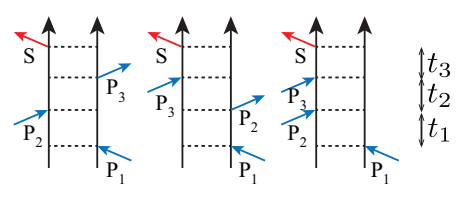

FIG. 1. Coherent 2D spectroscopic measurement of an interacting quantum many-body system. (a) System of spin- $1 / 2$ fermionic particles moving in a lattice. The two parameters of the Hubbard model are the hopping amplitude $J$ between neighboring sites and the on-site interaction $U$ between two particles at the same site. (b) Setup of the coherent 2D spectroscopic measurement. Three laser pulses successively interact with the system, and the light-emission signal induced by the polarization of the system is measured. (c) Variance $\Delta n$ of the number of particles at a single site in the quantum manybody ground state of the system. Here the number of lattice sites is $N=6$, the filling factor is one-half, i.e., the number of particles is equal to the number of sites, the total magnetization is zero, and the periodic boundary condition is applied. (d) Double-sided Feynman diagrams for the light-matter interaction processes involved in the rephasing (photon echo) signal.

diagonal/off-diagonal peaks in the coherent 2D spectrum represent processes with equal/unequal excitation and emission frequencies. Importantly, off-diagonal peaks can emerge only if the two transitions associated with the optical excitation and emission are coupled to each other [38-40]. However, notably, this kind of coherent coupling between two transitions is purely at the level of single-body physics, whereas the much more complex quantum many-body physics is investigated in this study.

The major challenge in the application of 2D spectroscopy to complex quantum many-body systems is that the interpretation of the spectrum typically relies on a detailed theoretical investigation in order to disentangle various contributions. In this work we aim to identify unique signatures that allow us to characterize many-body interactions in correlated systems over a broad range of interaction strength. We calculate the coherent 2D spectrum of an interacting quantum many-body system of spin-1/2 fermions [see Figs. 1(a) and 1(b)], which can be, for example, electrons moving in a crystal lattice or ultracold atoms/molecules moving in an artificial lattice. For the minimum two-site system, we observe that a system of noninteracting particles would display a 2D spectrum with only peaks lying on the diagonal axis $\omega_{1}=-\omega_{3}$. This is attributed to the fact that the quasimomentum of the particles is a good quantum number in the absence of interparticle interaction. Moreover, because of the conservation of momentum in the light-matter interaction, two optical transitions with different quasimomenta are not coupled to each other. In contrast, if the interaction between particles is sufficiently strong, off-diagonal peaks emerge in the $2 \mathrm{D}$ spectrum. In an interacting quantum many-body system, quasimomentum states are no longer energy eigenstates of the system. Consequently, two transitions with different optical excitation and emission frequencies can be effectively coupled to each other, leading to the emergence of off-diagonal peaks. However, if the interaction strength is increased further, when the system approaches the strongly interacting limit, in which exactly one particle is localized at each lattice site as the hopping of particles between neighboring sites becomes energetically unfavorable, all the peaks in the $2 \mathrm{D}$ spectrum coalesce into a single diagonal peak at the frequency of the excitation band. Consequently, coherent 2D spectroscopy can be used to investigate the entire range of interaction strength from weak- to strong-interaction regimes. We also investigate the coherent $2 \mathrm{D}$ spectrum of the system when the ratio of the interaction energy to the kinetic energy varies with time. The evolution of the $2 \mathrm{D}$ spectrum as a function of the time delay $t_{2}$ between the second and third pulses can provide us with important information on the ultrafast time variation of the interaction and the accompanying dynamics of the system. Notably, in contrast to other schemes [41,42], our proposed method to probe the quantum many-body interaction does not require single-site addressability, and thus can be suitable for studying ultracold atoms and ions as well as electronic systems.

\section{SYSTEM}

Consider the Hubbard model of a system of spin-1/2 fermionic particles moving in a lattice, whose Hamiltonian for the ground-state band is given by

$$
\hat{H}_{\mathrm{g}}=-J \sum_{\langle i, j\rangle} \sum_{\sigma=\uparrow, \downarrow}\left(\hat{c}_{i, \sigma}^{\dagger} \hat{c}_{j, \sigma}+\text { H.c. }\right)+U \sum_{j} \hat{n}_{j, \uparrow} \hat{n}_{j, \downarrow},
$$

where $\hat{c}_{j, \sigma}$ denotes the annihilation operator of a particle with spin $\sigma$ located at the $j$ th site, and $\hat{n}_{j, \sigma}=\hat{c}_{j, \sigma}^{\dagger} \hat{c}_{j, \sigma}$ is the particle number operator. The parameters $J$ and $U$ represent the hopping amplitude of the particle between neighboring sites and the on-site interaction between two particles located at the same site, respectively. Here $\langle\cdots\rangle$ denotes a pair of nearest neighboring sites, and H.c. stands for Hermitian conjugate. Here we restrict our consideration to the case that the number of particles is equal to the number of lattice sites. Furthermore, the total magnetization of the system is zero, i.e., the number of particles with spin-up is equal to that of particles with spin-down.

For a weakly interacting system with $|J| \gg U$, the motions of particles are almost independent of one another (except for the Pauli exclusive principle) and their wave functions are delocalized over different sites in the lattice. In contrast, in the strongly interacting limit $|J| \ll U$, the system would be in the Mott insulating phase, where each lattice site is occupied by exactly one particle as the hopping of particles between neighboring sites is energetically unfavorable. This transition is reflected by the change in the variance $\Delta n=\left\langle\psi_{0}\left|\hat{n}_{j}^{2}\right| \psi_{0}\right\rangle$ of the number of particles at a single site for the quantum many-body ground state $\left|\psi_{0}\right\rangle$. Figure $1(\mathrm{c})$ shows $\Delta n$ as a function of $U /|J|$ for a system of $N=6$ particles in a onedimensional lattice. Here $\hat{n}_{j}=\hat{n}_{j, \uparrow}+\hat{n}_{j, \downarrow}$. The variance is maximum for $U=0$, at which $\Delta n=1.5$; it decreases with 
increasing interaction strength and approaches $\Delta n=1$ for a sufficiently strong interaction.

We consider a one-dimensional system with the periodic boundary condition. As for the optical transitions caused by the light-matter interactions with the laser pulses in coherent 2D spectroscopy, we consider a Hubbard model with two energy bands: the ground-state and excited-state bands. The total Hamiltonian of the system is given by

$$
\begin{aligned}
\hat{H}= & -\sum_{j=0}^{N-1} \sum_{\sigma=\uparrow, \downarrow}\left(J_{\mathrm{g}} \hat{c}_{\mathrm{g}, j+1, \sigma}^{\dagger} \hat{c}_{\mathrm{g}, j, \sigma}+J_{\mathrm{e}} \hat{c}_{\mathrm{e}, j+1, \sigma}^{\dagger} \hat{c}_{\mathrm{e}, j, \sigma}+\text { H.c. }\right) \\
& +\sum_{j=0}^{N-1} \sum_{\alpha \neq \beta} U_{\alpha \beta} \hat{n}_{j, \alpha} \hat{n}_{j, \beta}+\epsilon_{\mathrm{eg}} \sum_{j=0}^{N-1} \sum_{\sigma=\uparrow, \downarrow} \hat{c}_{\mathrm{e}, j, \sigma}^{\dagger} \hat{c}_{\mathrm{e}, j, \sigma}, \quad \text { (2) }
\end{aligned}
$$

where $\alpha, \beta=(\mathrm{g} / \mathrm{e} ; \uparrow / \downarrow)$. Here $J_{\mathrm{g}}$ and $J_{\mathrm{e}}$ denote the hopping amplitudes of particles between neighboring sites in the ground and excited bands, respectively. Owing to the difference in the spatial confinement of the wave function of the particle at the lattice sites between the two bands, $J_{\mathrm{g}} \neq J_{\mathrm{e}}$. In general, the on-site interaction should also depend on the spins of the particles and on whether they are in the ground or excited band; however, for simplicity, we assume that the on-site interaction is characterized by a single variable parameter, namely $U_{\alpha \beta}=U$. The periodic boundary condition indicates that $j=N$ is equivalent to $j=0$. In the two-band Hubbard-model Hamiltonian (2), we ignored the Hund-type interaction, which is often included in the models of multiorbital electronic systems but would vanish if the excited band is the lowest-energy orbital of atoms in an electronic excited state such as in the experiment of Ref. [14]. The neglect of Hund-type interaction does not qualitatively change the conclusions of this paper.

As the wavelengths of the laser pulses are typically much larger than the lattice constant of the crystal lattice for electrons, the lasers' electric fields can be considered homogeneous over a large number of lattice sites. A similar nanoscale artificial lattice for ultracold atoms can be realized by using nanoplasmonic systems [43], photonic crystals [44], time-periodic modulations [45], and superconductors [46]. In this case, at a length scale that is small compared to the wavelengths of the lasers but large compared to the lattice constant, the phase $e^{i \mathbf{k} \cdot \mathbf{r}}$ of a laser can be considered constant, corresponding to zero momentum of photon. The GoppertMayer gauge transformation can then be applied to express the light-matter interaction Hamiltonian in the form of $\mathbf{E}(t) \cdot \hat{\mathbf{D}}$ [47], where the dipole moment operator $\hat{\mathbf{D}}$ is given in the two-band Hubbard model, i.e., expanded in the Wannier orbital basis, as $\hat{\mathbf{D}}=\sum_{i, j=0}^{N-1} \sum_{l, l^{\prime}=\mathrm{g}, \mathrm{e}} \sum_{\sigma=\uparrow, \downarrow} \hat{c}_{l, i, \sigma}^{\dagger} \mu_{l, i, l^{\prime}, j} \hat{c}_{l^{\prime}, j, \sigma}$. Here $\mu_{l, i, l^{\prime}, j}=\left\langle l, i|\hat{\mathbf{D}}| l^{\prime}, j\right\rangle$ is the matrix element of the dipole moment operator for the $\left(l^{\prime}, j\right) \rightarrow(l, i)$ transition. Note that here we did not use the so-called multicenter Power-ZienauWoolley transformation [48], which is appropriate for the more general case of a nonuniform electromagnetic field, and for which the Peierls phase [49] emerges in addition to the dipole interaction. It is also noteworthy that even though the gauge invariance would break down by a truncation of the full Hilbert space to a few lowest-energy bands, the difference between results obtained by using different gauges is only significant in the ultrastrong coupling regime, where the light-matter interaction is comparable in magnitude with the transition frequency of the system [50], as opposed to the typically weak-coupling regime of spectroscopic measurements under consideration. At larger length scales, the phases of lasers are imprinted into the phase of the system's nonlinear polarization, leading to the phase matching condition which can be interpreted as the conservation of momentum in the coupled light-matter system [51]. The optical signal $S(t)$ measured via coherent 2D spectroscopy is proportional to $i P^{(3)}(t)$, where $P^{(3)}(t)$ is the time-dependent third-order polarization of the system. It can be expressed as a convolution of the third-order response function $R^{(3)}\left(\tau_{1}, \tau_{2}, \tau_{3}\right)$ and the electric fields of the lasers $[22,28]$

$$
\begin{aligned}
P^{(3)}(t)= & \int_{0}^{\infty} d \tau_{1} \int_{0}^{\infty} d \tau_{2} \int_{0}^{\infty} d \tau_{3} R^{(3)}\left(\tau_{1}, \tau_{2}, \tau_{3}\right) \\
& \times E\left(t-\tau_{3}\right) E\left(t-\tau_{3}-\tau_{2}\right) E\left(t-\tau_{3}-\tau_{2}-\tau_{1}\right) .
\end{aligned}
$$

In the impulsive limit of the laser pulses, where the electric field is given by a sum of three Dirac's delta functions, the polarization is proportional to the nonlinear response function $R^{(3)}\left(t_{1}, t_{2}, t_{3}\right)$, where $t_{1}, t_{2}$, and $t_{3}$ are the time intervals between the laser pulses and the emitted signal (see Fig. 1). The nonlinear response function and emitted signal are generated by various processes, each of which involves four interactions between light and matter. These processes can be grouped into three categories according to the direction of the emitted signal: rephasing, nonrephasing, and double quantum coherence. The three types of signals can generally provide different types of information about the energy levels and dynamics of the system. The double quantum coherence signal relies on the difference in energy between the transition from zero to one excitation and that from one to two excitations, and has been used to detect interaction between two specific transitions such as excitons in semiconductors [52] and transition lines in atomic vapors [53,54]. Note, however, that for the system of interacting particles moving in a lattice under consideration, the 2D spectra involves an infinite number of transitions, and therefore might make an interpretation of double quantum coherence signal much more difficult. In this study we investigate the rephasing signal as we concentrate on the effective coupling between transitions induced by the quantum many-body interaction. The rephasing or photon-echo signal is detected in the direction given by the vector $\mathbf{k}_{\mathrm{r}}=-\mathbf{k}_{1}+\mathbf{k}_{2}+\mathbf{k}_{3}$. The light-matter interactions for the processes included in the rephasing signal are illustrated by the double-sided Feynman diagram [Fig. 1(d)] The corresponding rephasing third-order response function $R_{\mathrm{r}}^{(3)}\left(t_{1}, t_{2}, t_{3}\right)$ can be expressed in terms of the Liouville-space operators as [22]

$$
\begin{aligned}
& R_{\mathrm{r}}^{(3)}\left(t_{1}, t_{2}, t_{3}\right) \\
& \quad=\left(\frac{i}{\hbar}\right)^{3} \operatorname{Tr}\left\{\hat{\mu}_{\leftarrow} \mathcal{G}\left(t_{3}\right) \hat{\mu}_{\rightarrow}^{\times} \mathcal{G}\left(t_{2}\right) \hat{\mu}_{\rightarrow}^{\times} \mathcal{G}\left(t_{1}\right) \hat{\mu}_{\leftarrow}^{\times} \hat{\rho}_{0}\right\},
\end{aligned}
$$


(a)

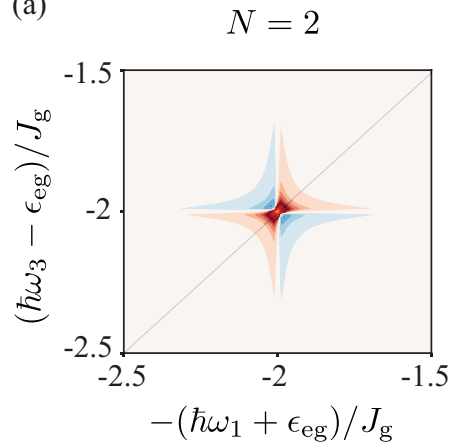

(b)



(c)

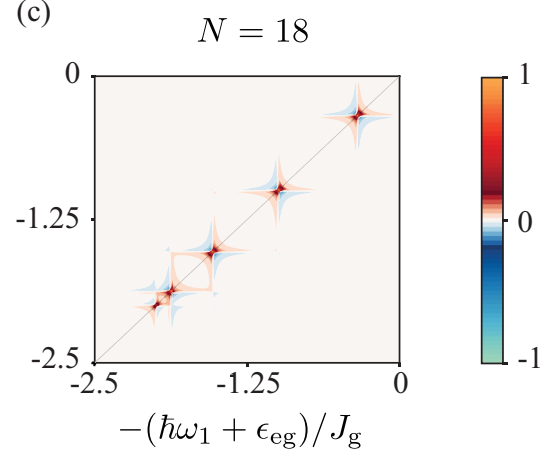

FIG. 2. Coherent 2D rephasing spectra of a system of noninteracting spin- $1 / 2$ fermionic particles with different lattice sizes. The number of lattice sites is $N=2$ in (a), $N=10$ in (b), and $N=18$ in (c). The spectral intensity is normalized by its maximum value and represented by the color scale. The frequencies $\omega_{1}$ and $\omega_{3}$ are normalized by the hopping amplitude $J_{\mathrm{g}}$ between neighboring sites of particles moving in the ground-state band. Here the origin of the $2 \mathrm{D}$ spectrum was shifted by the energy gap $\epsilon_{\mathrm{eg}}$ between the ground-state and excited-state bands. The diagonal axis $\omega_{3}=-\omega_{1}$ is shown as a guide for the eye.

where $\hat{\rho}_{0}=\left|\psi_{0}\right\rangle\left\langle\psi_{0}\right|$ is the density operator for the quantum many-body ground state of the system, and the transition

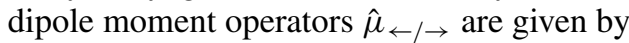

$$
\begin{aligned}
& \hat{\mu}_{\leftarrow}=\sum_{j=0}^{N-1} \sum_{\sigma=\uparrow, \downarrow} \mu_{\mathrm{ge}} \hat{c}_{\mathrm{g}, j, \sigma}^{\dagger} \hat{c}_{\mathrm{e}, j, \sigma}, \\
& \hat{\mu}_{\rightarrow}=\sum_{j=0}^{N-1} \sum_{\sigma=\uparrow, \downarrow} \mu_{\mathrm{eg}} \hat{c}_{\mathrm{e}, j, \sigma}^{\dagger} \hat{c}_{\mathrm{g}, j, \sigma} .
\end{aligned}
$$

Here $\mu_{\mathrm{eg}}$ is the transition dipole moment between the ground and excited bands, namely the interband transition, and $\mu_{\mathrm{ge}}=$ $\mu_{\mathrm{eg}}^{*}$ [51]. The intraband transition was neglected as the laser pulses are assumed to be far off resonant with that transition. The superoperators in Liouville space are defined as $\hat{\mu}^{\times} \hat{\rho}=$ $\hat{\mu} \hat{\rho}-\hat{\rho} \hat{\mu}$ and $\mathcal{G}(t) \hat{\rho}=e^{-i \hat{H} t / \hbar} \hat{\rho} e^{i \hat{H} t / \hbar}$, where $\hat{H}$ is the quantum many-body Hamiltonian of the system given in Eq. (2). A small dephasing rate $\kappa$ is introduced to account for the dephasing of the quantum coherence between the ground and excited states during the time intervals $t_{1}$ and $t_{3}$. The coherent $2 \mathrm{D}$ rephasing spectrum is obtained by making a Fourier transformation of the emitted signal $S\left(t_{1}, t_{2}, t_{3}\right)$ with respect to the time intervals $t_{1}$ and $t_{3}$,

$$
S\left(\omega_{1}, t_{2}, \omega_{3}\right)=\int_{0}^{\infty} d t_{1} \int_{0}^{\infty} d t_{3} e^{i\left(\omega_{1} t_{1}+\omega_{3} t_{3}\right)} S\left(t_{1}, t_{2}, t_{3}\right)
$$

In the following 2D spectra, the real part of $S\left(\omega_{1}, t_{2}, \omega_{3}\right)$ is plotted as a function of $-\omega_{1}$ and $\omega_{3}$.

We first consider a system of noninteracting particles, namely $U=0$. The relative signs and magnitudes of the hopping amplitudes $J_{\mathrm{g}}$ and $J_{\mathrm{e}}$ for the ground-state and excitedstate bands depend on the details of the particle's internal states and the lattice potentials. For example, if the excitedstate band is the lowest-energy orbital for atoms in an electronic excited state such as the ${ }^{1} S_{0} \rightarrow{ }^{3} P_{2}$ transition of $\mathrm{Yb}$ atom [14], $J_{\mathrm{g}}$ and $J_{\mathrm{e}}$ have the same sign. However, the relative signs and magnitudes of $J_{\mathrm{g}}$ and $J_{\mathrm{e}}$ do not qualitatively change the conclusions of this work. Here the system's parameters were considered as follows: $J_{\mathrm{e}} / J_{\mathrm{g}}=2$ and $\hbar \kappa / J_{\mathrm{g}}=0.01$ corresponding to a dephasing time of the picosecond order due to, for example, the electron-phonon coupling. The time delay was set to $t_{2}=0$. The coherent $2 \mathrm{D}$ rephasing spectra for different lattice sizes are shown in Fig. 2. It is evident that all the peaks lie on the diagonal axis $\omega_{3}=-\omega_{1}$. The number of peaks increases with the lattice size. In the thermodynamic limit $(N \rightarrow \infty)$, the signal in the $2 \mathrm{D}$ spectrum is the segment $\epsilon_{\text {eg }}-2\left(J_{\mathrm{e}}-J_{\mathrm{g}}\right) \leqslant \hbar \omega_{3}=-\hbar \omega_{1} \leqslant \epsilon_{\mathrm{eg}}$ of the diagonal axis. Note that throughout this paper the origin of $2 \mathrm{D}$ spectrum is shifted by the energy gap $\epsilon_{\mathrm{eg}}$ between the ground-state and excited-state bands, namely $\hbar \tilde{\omega}_{1}=\hbar \omega_{1}+\epsilon_{\text {eg }}$ and $\hbar \tilde{\omega}_{3}=$ $\hbar \omega_{3}-\epsilon_{\text {eg. }}$. Thus, the origin $\tilde{\omega}_{1}=\tilde{\omega}_{3}=0$ of the $2 \mathrm{D}$ spectrum corresponds to $\hbar \omega_{3}=-\hbar \omega_{1}=\epsilon_{\mathrm{eg}}$.

In the absence of interaction between particles, quasimomentum is a good quantum number. The single-particle energy eigenstates in the ground and excited bands are characterized by a wave vector $k$ (within the Brillouin zone) with the corresponding energy eigenvalues given by $\epsilon_{k}^{\mathrm{g}}=$ $-2 J_{\mathrm{g}} \cos (2 \pi k / N)$ and $\epsilon_{k}^{\mathrm{e}}=\epsilon_{e g}-2 J_{\mathrm{e}} \cos (2 \pi k / N)$ with $k=$ $-N / 2, \ldots, N / 2$. Owing to the conservation of momentum in the light-matter interaction, optical transitions can only occur between pairs of single-particle energy eigenstates in the ground and excited bands with the same wave vector. As these transitions are not coupled to one another, only diagonal peaks appear in the coherent $2 \mathrm{D}$ rephasing spectrum. With the half-filling factor, the quantum many-body ground state of the system contains all the single-particle energy eigenstates of $\hat{H}_{\mathrm{g}}$ with the wave vectors $-N / 4 \leqslant k \leqslant N / 4$ (for $J_{\mathrm{g}}>0$ ). The corresponding transition energy satisfies $\epsilon_{e g}-2\left(J_{\mathrm{e}}-J_{\mathrm{g}}\right) \leqslant$ $\epsilon_{k}^{\mathrm{e}}-\epsilon_{k}^{\mathrm{g}} \leqslant \epsilon_{e g}$.

Next, we consider a system of interacting particles, namely $U>0$ (repulsive interaction). As the computational cost increases exponentially with the system size, we restrict the computation to a small system of $N=2$ lattice sites. The coherent 2D rephasing spectra for different interaction strengths (normalized by the hopping amplitude $J_{\mathrm{g}}$ ) are shown in Fig. 3. The other parameters of the system are the same as in the case of noninteracting particles. As the interparticle interaction becomes stronger, an off-diagonal peak starts to emerge at $U / J_{\mathrm{g}} \simeq 5$ in addition to the diagonal peak. The off-diagonal peak persists up to an interaction of $U / J_{\mathrm{g}} \simeq 100$. Finally, at the strongly interacting limit $U / J_{\mathrm{g}} \simeq 1000$, all the peaks coalesce into a single diagonal peak at the origin of the $2 \mathrm{D}$ spectrum. In the presence of the interparticle interaction, the quantum many-body ground state cannot be expressed by a 
(a)

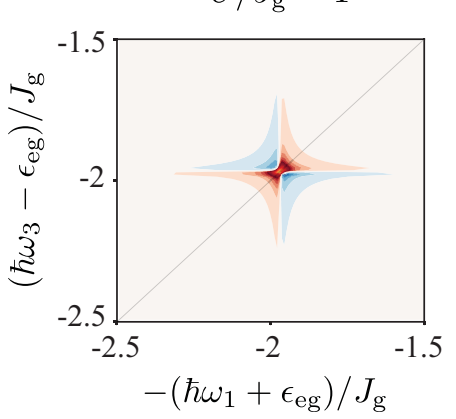

(d)

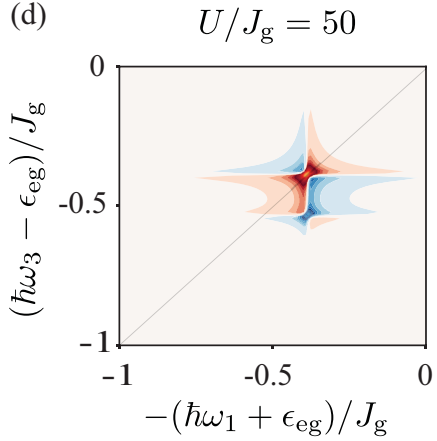

(b)



(e)

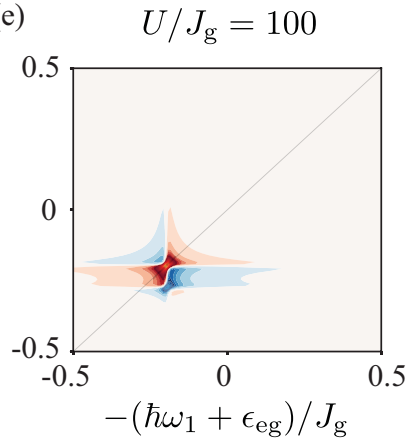

(c)

$U / J_{\mathrm{g}}=10$

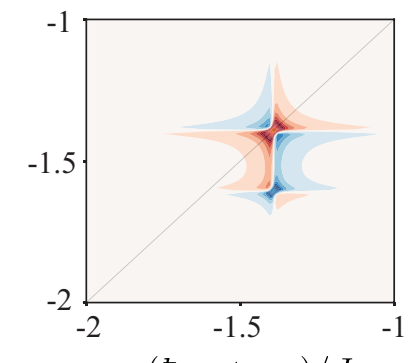

(f)

$$
-\left(\hbar \omega_{1}+\epsilon_{\mathrm{eg}}\right) / J_{\mathrm{g}}
$$

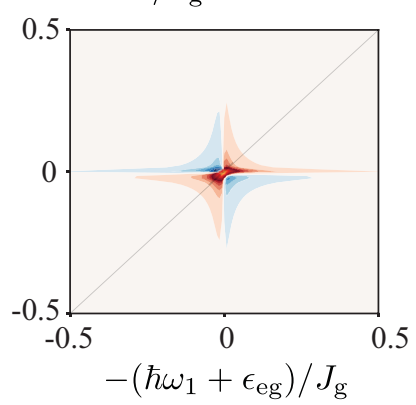

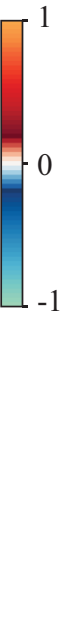

FIG. 3. Coherent 2D rephasing spectra of a system of interacting spin- $1 / 2$ fermionic particles with different interaction strengths. Here the interaction strength $U$ is normalized by the hopping amplitude $J_{\mathrm{g}}$ and the number of lattice sites is $N=2$.

collection of single-particle states. Consequently, the optical transitions become effectively coupled to one another, leading to the emergence of off-diagonal peaks. In the strongly interacting limit, the quantum many-body ground state is in the Mott insulating phase with each lattice site being occupied by exactly one particle. Similar to the noninteracting limit, there is no entanglement between the particles in the limit of Mott insulator as the total wave function is a product of localized single-particle states. The optical excitation of each particle occurs locally with an excitation energy equal to $\epsilon_{\text {eg }}$. This results in a single diagonal peak at the origin of the $2 \mathrm{D}$ spectrum. In the thermodynamic limit $(N \rightarrow \infty)$ we conjecture that as the interaction between particles gets stronger, the signal in 2D spectrum expands away from the diagonal axis, and finally at the Mott-insulator limit, it shrinks to a single diagonal peak at the origin of the spectrum corresponding to the energy gap between two bands. The expansion of the $2 \mathrm{D}$ spectral signal away from the diagonal axis is a consequence of the effective coupling between transitions with different frequencies via the quantum many-body interaction. Since the propagation of the coupling is done via the hopping of particles between lattice sites, the displacement of the signal from the diagonal axis should be a function of both the interaction and the hopping amplitude. The displacement vanishes in both the weakly interacting limit $U / J_{\mathrm{g}} \rightarrow 0$ and the Mott-insulator limit $U / J_{\mathrm{g}} \rightarrow \infty$, and in the thermodynamic limit, when $U$ and $J_{\mathrm{g}}$ are comparable in magnitude, the displacement is expected to have this order of magnitude. For electronic systems, both the Coulomb interaction and the hopping amplitude are of the order of an electron volt. For a system of ultracold atoms in an artificial lattice, the hopping amplitude is of the order of the recoil energy $E_{\mathrm{R}}=h^{2} /(8 m a)$, where $h$ is the Planck's constant, $m$ is the mass of the atom, and $a$ is the lattice constant. For a nanoscale artificial lattice, the recoil energy and in turn the hopping amplitude is of the order of GHz. Such a GHz-order-of-magnitude displacement of the 2D spectral signal from the diagonal axis can be well measured with the currently attainable frequency resolution of $2 \mathrm{D}$ coherent spectroscopy [55]. An investigation of the displacement of 2D spectral signal from the diagonal axis as a function of the ratio $U / J_{\mathrm{g}}$ would provide valuable information about the metal-to-Mott-insulator phase transition.

Finally, we consider the case of a time-varying interaction $U(t)$ and investigate the coherent 2D spectrum as a function of the time delay $t_{2}$. The study of dynamics in interacting quantum many-body systems is of high importance in modern physics as much less is understood about nonequilibrium than equilibrium properties. By making a time-dependent interaction, the system can be prepared in a nonequilibrium state and the ensuing dynamics can be studied. It is a highly nontrivial and intriguing issue to investigate nonequilibrium dynamics across a quantum phase transition such as the metalto-Mott-insulator transition where universal behaviors might be observed [56]. In ultracold atomic systems, the interaction strength can be varied by using, for example, the Feshbach resonance technique [57], or the ratio $U / J_{\mathrm{g}}$ of the interaction to the hopping amplitude can be varied by changing the depth of the lattice potential. In electronic systems, the interaction between electrons might be effectively manipulated by applying a pressure or by driving the system with a laser. If the interaction is switched off abruptly at time $\tau$ after the incidence of the first laser pulse, i.e., $U(t)=U_{0}$ for $t \leqslant \tau$ 
(a)

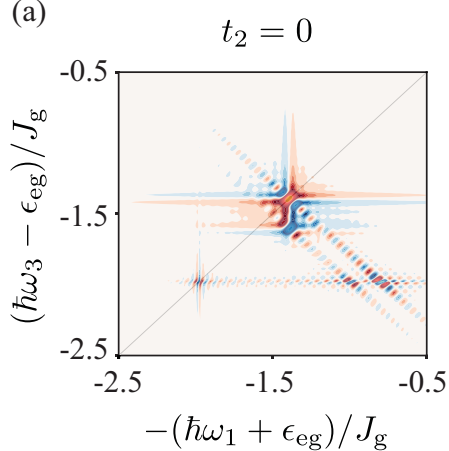

(d)

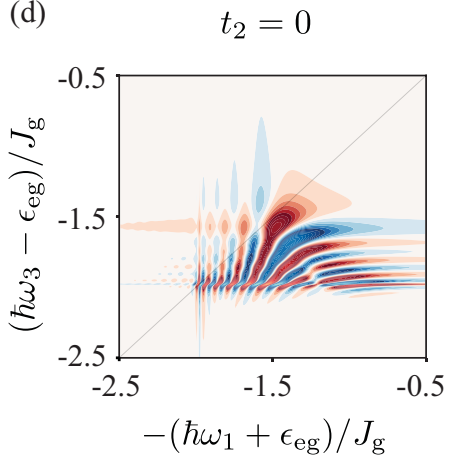

(b)

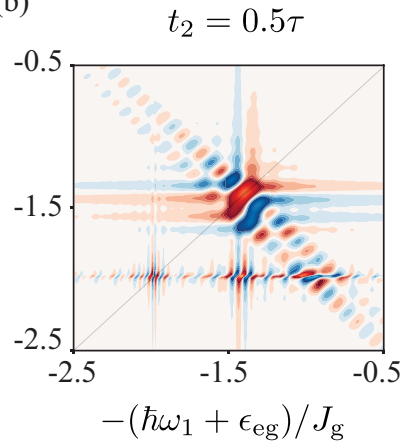

(e)

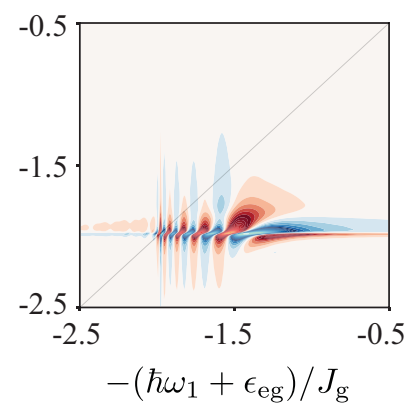

(c)

(c) $t_{2}=\tau$

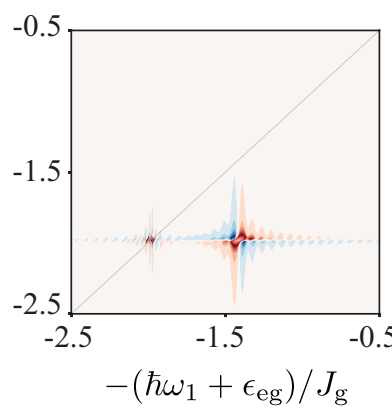

(f)

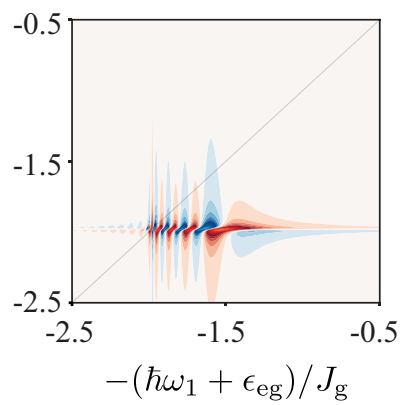

FIG. 4. Coherent 2D rephasing spectra of a system of interacting spin- $1 / 2$ fermionic particles as a function of the time delay $t_{2}$ between the second and third laser pulses. (a)-(c) The interaction strength is abruptly switched off from $U / J_{\mathrm{g}}=10$ to $U=0$ at time $\tau=100 \hbar / J_{\mathrm{g}}$. (d) - (f) The interaction strength is switched off steadily over the time interval of $\tau$.

and $U(t)=0$ for $t>\tau$, the coherent 2D spectra for different values of the time delay $t_{2}$ are shown in Figs. 4(a)-4(c). Here the initial interaction strength is $U_{0} / J_{\mathrm{g}}=10$, the switching time is $\tau J_{\mathrm{g}}=100 \hbar$, and the other parameters of the system are the same as in the case of a time-independent interaction. The $2 \mathrm{D}$ spectrum is observed to change from $t_{2}=0$ to $t_{2}=\tau$, and subsequently, it remains almost unchanged. The 2D spectrum at $t_{2}=0$ shows the pair of a diagonal peak at $\hbar \omega_{1}=-\hbar \omega_{3} \simeq$ $1.4 J_{\mathrm{g}}$ and an off-diagonal peak at $\hbar \omega_{1} \simeq 1.4 \mathrm{~J}_{\mathrm{g}}, \hbar \omega_{3} \simeq-1.6 \mathrm{~J}_{\mathrm{g}}$ that also appear in the $2 \mathrm{D}$ spectrum for constant $U=U_{0}$ [see Fig. 3(c)]. These peaks are, however, extended along the diagonal direction of $\omega_{1}=\omega_{3}$ to $\hbar \omega_{3}=-2 J_{\mathrm{g}}$, which is the emission frequency for the single diagonal peak in the 2D spectrum for constant $U=0$ [see Fig. 3(a) for the 2D spectrum for constant $U / J_{\mathrm{g}}=1$ which is close to that for constant $U=0]$. The spectrum also contains a small diagonal peak at $\hbar \omega_{1}=-\hbar \omega_{3}=2 J_{\mathrm{g}}$, which is the peak in the $2 \mathrm{D}$ spectrum for constant $U=0$. The $2 \mathrm{D}$ spectrum changes with the variable time delay. At $t_{2}=\tau$ it is composed of a small diagonal peak at $\hbar \omega_{1}=-\hbar \omega_{3}=2 J_{\mathrm{g}}$ as at $t_{2}=0$, an off-diagonal peak at $\hbar \omega_{1} \simeq 1.4 J_{\mathrm{g}}, \hbar \omega_{3}=-2 J_{\mathrm{g}}$, i.e., the same excitation frequency as the peaks in the $2 \mathrm{D}$ spectrum for constant $U=U_{0}$ and the same emission frequency as the peak in the $2 \mathrm{D}$ spectrum for constant $U=0$, and a connection between them. Therefore, the $2 \mathrm{D}$ spectrum as a function of the time delay $t_{2}$ contains information of the 2D spectra associated with the initial and final values of the interaction as well as the time evolution in between.

If the interaction is switched off steadily over a time period of $\tau$ after the incidence of the first laser pulse, i.e., $U(t)=U_{0}(1-t / \tau)$ for $t \leqslant \tau$ and $U(t)=0$ for $t>\tau$, the coherent 2D spectra for different time delays are shown in Figs. 4(d)-4(f). A fringe pattern emerges in the 2D spectrum, which should be attributed to the continuous time variation of the interaction strength. The spectrum changes gradually with a variable time delay. At $t_{2}=\tau$, it consists of an array of peaks distributed on the segment between $\hbar \omega_{1} \simeq 1.4 J_{\mathrm{g}}$ and $\hbar \omega_{1}=2 J_{\mathrm{g}}$, i.e., the excitation frequencies of the peaks in the 2D spectra for constant $U=U_{0}$ and for constant $U=0$, respectively, of the horizontal line $\hbar \omega_{3}=-2 J_{\mathrm{g}}$, i.e., the emission frequency of the peak in the $2 \mathrm{D}$ spectrum for constant $U=0$.

\section{CONCLUSION}

We investigated the coherent $2 \mathrm{D}$ spectrum of an interacting quantum many-body system of spin- $1 / 2$ fermions moving in a lattice, for example, electronic and ultracold atomic systems. In the weakly interacting limit, the 2D rephasing spectrum manifests itself as a segment on the diagonal axis because different optical transitions are not coupled to one another. In contrast, if the interaction between particles is sufficiently strong, the signal of $2 \mathrm{D}$ spectrum expands away from the diagonal axis, which can be used as a direct probe of a non-negligible interaction. It is attributed to the fact that different optical transitions can be effectively coupled to one another in the presence of interparticle interaction. Notably, effective coupling is induced by the quantum many-body interaction between particles as opposed to the conventional coherent coupling between two transitions at the level of single-body physics. As the interaction strength is increased further, when the system approaches the Mott 
insulating phase in the strongly interacting limit, the $2 \mathrm{D}$ spectrum shrinks to a single diagonal peak at the origin of the spectrum, i.e., at the transition frequency between the ground-state and excited-state energy bands. Moreover, when the interaction is time dependent, the information of its time variation can be obtained from the evolution of the coherent 2D spectrum as a function of the time delay between the second and third pulses. The results of this study demonstrate the potential of coherent multidimensional spectroscopy for studying quantum many-body interactions and ultrafast dynamics in various kinds of strongly correlated systems. Another advantage of using ultrafast 2D spectroscopy to study coherent quantum many-body interactions is that the effects of incoherent processes occurring at longer timescales can be separated. A quantitative characterization of the metal-Mottinsulator phase transition point by the coherent $2 \mathrm{D}$ spectrum is, however, still an interesting open question, which requires a numerical or experimental study of a large-size system of interacting particles.

\section{ACKNOWLEDGMENTS}

N.T.P. would like to thank Akihito Ishizaki and Yuta Fujihashi for discussion in preparing the manuscript. This work was supported by JSPS KAKENHI Grant No. 19K14638 (N.T.P.). The computations were performed using Research Center for Computational Science, Okazaki, Japan.
[1] L. D. Landau, The theory of a Fermi liquid, Zh. Eksp. Teor. Fiz. 30, 1058 (1956) [Sov. Phys. JETP 3, 920 (1957)].

[2] S. Tomonaga, Remarks on Bloch's method of sound waves applied to many-fermion problems, Prog. Theor. Phys. 5, 544 (1950).

[3] J. M. Luttinger, An exactly soluble model of a many-fermion system, J. Math. Phys. 4, 1154 (1963).

[4] G. R. Stewart, Non-Fermi-liquid behavior in $d$ - and $f$-electron metals, Rev. Mod. Phys. 73, 797 (2001).

[5] N. F. Mott, Metal-insulator transition, Rev. Mod. Phys. 40, 677 (1968).

[6] M. Imada, A. Fujimori, and Y. Tokura, Metal-insulator transitions, Rev. Mod. Phys. 70, 1039 (1998).

[7] A. J. Leggett, Quantum Liquids: Bose Condensation and Cooper Pairing in Condensed-Matter Systems (Oxford University Press, New York, 2006).

[8] I. Bloch, J. Dalibard, and W. Zwerger, Many-body physics with ultracold gases, Rev. Mod. Phys. 80, 885 (2008).

[9] I. Bloch, J. Dalibard, and S. Nascimbene, Quantum simulations with ultracold quantum gases, Nat. Phys. 8, 267 (2012).

[10] C. Gross and I. Bloch, Quantum simulations with ultracold atoms in optical lattices, Science 357, 995 (2017).

[11] S. A. Moses, J. P. Covey, M. T. Miecnikowski, D. S. Jin, and J. Ye, New frontiers for quantum gases of polar molecules, Nat. Phys. 13, 13 (2017).

[12] J. L. Bohn, A. M. Rey, and J. Ye, Cold molecules: Progress in quantum engineering of chemistry and quantum matter, Science 357, 1002 (2017).

[13] G. K. Campbell, J. Mun, M. Boyd, P. Medley, A. E. Leanhardt, L. Marcassa, D. E. Pritchard, and W. Ketterle, Imaging the Mott insulator shells by using atomic clock shifts, Science 313, 649 (2006).

[14] S. Kato, K. Inaba, S. Sugawa, K. Shibata, R. Yamamoto, M. Yamashita, and Y. Takahashi, Laser spectroscopic probing of coexisting superfluid and insulating states of an atomic BoseHubbard system, Nat. Commun. 7, 11341 (2016).

[15] F. Krausz and M. Ivanov, Attosecond physics, Rev. Mod. Phys. 81, 163 (2009).

[16] D. Fausti, R. I. Tobey, N. Dean, S. Kaiser, A. Dienst, M. C. Hoffmann, S. Pyon, T. Takayama, H. Takagi, and A. Cavalleri, Light-induced superconductivity in a stripe-ordered cuprate, Science 331, 189 (2011).
[17] A. Cavalleri, Photo-induced superconductivity, Contemp. Phys. 59, 31 (2018).

[18] A. V. Kimel, A. Kirilyuk, P. A. Usachev, R. V. Pisarev, A. M. Balbashov, and T. Rasing, Ultrafast non-thermal control of magnetization by instantaneous photomagnetic pulses, Nature (London) 435, 655 (2005).

[19] A. Kirilyuk, A. V. Kimel, and T. Rasing, Ultrafast optical manipulation of magnetic order, Rev. Mod. Phys. 82, 2731 (2010).

[20] Y. H. Wang, H. Steinberg, P. Jarillo-Herrero, and N. Gedik, Observation of Floquet-Bloch states on the surface of a topological insulator, Science 342, 453 (2013).

[21] J. W. McIver, B. Schulte, F.-U. Stein, T. Matsuyama, G. Jotzu, G. Meier, and A. Cavalleri, Light-induced anomalous Hall effect in graphene, Nat. Phys. 16, 38 (2020).

[22] S. Mukamel, Principles of Nonlinear Optical Spectroscopy (Oxford University Press, New York, 1995).

[23] S. Mukamel, Multidimensional femtosecond correlation spectroscopies of electronic and vibrational excitations, Annu. Rev. Phys. Chem. 51, 691 (2000).

[24] D. M. Jonas, Two-dimensional femtosecond spectroscopy, Annu. Rev. Phys. Chem. 54, 425 (2003).

[25] M. Cho, Coherent two-dimensional optical spectroscopy, Chem. Rev. 108, 1331 (2008).

[26] M. Cho, Two-Dimensional Optical Spectroscopy (CRC, Taylor \& Francis Group, Boca Raton, FL, 2009).

[27] D. Abramavicius, B. Palmieri, D. V. Voronine, F. Sanda, and S. Mukamel, Coherent multidimensional optical spectroscopy of excitons in molecular aggregates; quasiparticle versus supermolecule perspectives, Chem. Rev. 109, 2350 (2009).

[28] P. Hamm and M. Zanni, Concepts and Methods of 2D Infrared Spectroscopy (Cambridge University Press, New York, 2011).

[29] S. T. Cundiff, T. Zhang, A. D. Bristow, D. Karaiskaj, and X. Dai, Optical two-dimensional Fourier transform spectroscopy of semiconductor quantum wells, Acc. Chem. Res. 42, 1423 (2009).

[30] G. S. Schlau-Cohen, A. Ishizaki, and G. R. Fleming, Twodimensional electronic spectroscopy and photosynthesis: Fundamentals and applications to photosynthetic light-harvesting, Chem. Phys. 386, 1 (2011).

[31] W. Kuehn, K. Reimann, M. Woerner, T. Elsaesser, and R. Hey, Two-dimensional terahertz correlation spectra of electronic 
excitations in semiconductor quantum wells, J. Phys. Chem. B 115, 5448 (2011).

[32] M. Woerner, W. Kuehn, P. Bowlan, K. Reimann, and T. Elsaesser, Ultrafast two-dimensional terahertz spectroscopy of elementary excitations in solids, New J. Phys. 15, 025039 (2013).

[33] J. Lu, X. Li, H. Y. Hwang, B. K. Ofori-Okai, T. Kurihara, T. Suemoto, and K. A. Nelson, Coherent Two-Dimensional Terahertz Magnetic Resonance Spectroscopy of Collective Spin Waves, Phys. Rev. Lett. 118, 207204 (2017).

[34] Y. Wan and N. P. Armitage, Resolving Continua of Fractional Excitations by Spinon Echo in THz 2D Coherent Spectroscopy, Phys. Rev. Lett. 122, 257401 (2019).

[35] W. Choi, K. H. Lee, and Y. B. Kim, Theory of Two-Dimensional Nonlinear Spectroscopy for the Kitaev Spin Liquid, Phys. Rev. Lett. 124, 117205 (2020).

[36] F. Mahmood, D. Chaudhuri, S. Gopalakrishnan, R. Nandkishore, and N. P. Armitage, Observation of a marginal Fermi glass, Nat. Phys. 17, 627 (2021).

[37] F. Novelli, J. O. Tollerud, D. Prabhakaran, and J. A. Davis, Persistent coherence of quantum superpositions in an optimally doped cuprate revealed by 2D spectroscopy, Sci. Adv. 6, eaaw9932 (2020).

[38] X. Dai, A. D. Bristow, D. Karaiskaj, and S. T. Cundiff, Two-dimensional Fourier-transform spectroscopy of potassium vapor, Phys. Rev. A 82, 052503 (2010).

[39] M. Khalil, N. Demirdoven, and A. Tokmakoff, Obtaining Absorptive Line Shapes in Two-Dimensional Infrared Vibrational Correlation Spectra, Phys. Rev. Lett. 90, 047401 (2003).

[40] J. Zheng, K. Kwak, J. Asbury, X. Chen, I. R. Piletic, and M. D. Fayer, Ultrafast dynamics of solute-solvent complexation observed at thermal equilibrium in real time, Science 309, 1338 (2005).

[41] M. Gessner, F. Schlawin, H. Haffner, S. Mukamel, and A. Buchleitner, Nonlinear spectroscopy of controllable many-body quantum systems, New J. Phys. 16, 092001 (2014).

[42] F. Schlawin, M. Gessner, S. Mukamel, and A. Buchleitner, Nonlinear spectroscopy of trapped ions, Phys. Rev. A 90, 023603 (2014).

[43] M. Gullans, T. G. Tiecke, D. E. Chang, J. Feist, J. D. Thompson, J. I. Cirac, P. Zoller, and M. D. Lukin, Nanoplasmonic Lattices for Ultracold Atoms, Phys. Rev. Lett. 109, 235309 (2012).

[44] A. Gonzalez-Tudela, C.-L. Hung, D. E. Chang, J. I. Cirac, and H. J. Kimble, Subwavelength vacuum lattices and atomatom interactions in two-dimensional photonic crystals, Nat. Photonics 9, 320 (2015).
[45] S. Nascimbene, N. Goldman, N. R. Cooper, and J. Dalibard, Dynamic Optical Lattices of Subwavelength Spacing for Ultracold Atoms, Phys. Rev. Lett. 115, 140401 (2015).

[46] O. Romero-Isart, C. Navau, A. Sanchez, P. Zoller, and J. I. Cirac, Superconducting Vortex Lattices for Ultracold Atoms, Phys. Rev. Lett. 111, 145304 (2013).

[47] C. Cohen-Tannoudji, J. Dupont-Roc, and G. Grynberg, Photons and Atoms: Introduction to Quantum Electrodynamics (Wiley, Weinheim, 2004).

[48] J. Li, D. Golez, G. Mazza, A. J. Millis, A. Georges, and M. Eckstein, Electromagnetic coupling in tight-binding models for strongly correlated light and matter, Phys. Rev. B 101, 205140 (2020).

[49] R. Peierls, Zur Theorie des Diamagnetismus von Leitungselektronen, Z. Physik 80, 763 (1933).

[50] D. De Bernardis, P. Pilar, T. Jaako, S. De Liberato, and P. Rabl, Breakdown of gauge invariance in ultrastrong-coupling cavity QED, Phys. Rev. A 98, 053819 (2018).

[51] See Supplemental Material at http://link.aps.org/supplemental/ 10.1103/PhysRevB.104.115105 for discussions on light-matter coupling and optical signal in coherent two-dimensional spectroscopy.

[52] D. Karaiskaj, A. D. Bristow, L. Yang, X. Dai, R. P. Mirin, S. Mukamel, and S. T. Cundiff, TwoQuantum Many-Body Coherences in Two-Dimensional Fourier-Transform Spectra of Exciton Resonances in Semiconductor Quantum Wells, Phys. Rev. Lett. 104, 117401 (2010).

[53] X. Dai, M. Richter, H. Li, A. D. Bristow, C. Falvo, S. Mukamel, and S. T. Cundiff, Two-Dimensional Double-Quantum Spectra Reveal Collective Resonances in an Atomic Vapor, Phys. Rev. Lett. 108, 193201 (2012).

[54] B. Lomsadze and S. T. Cundiff, Frequency-Comb Based Double-Quantum Two-Dimensional Spectrum Identifies Collective Hyperfine Resonances in Atomic Vapor Induced by Dipole-Dipole Interactions, Phys. Rev. Lett. 120, 233401 (2018).

[55] B. Lomsadze and S. T. Cundiff, Frequency combs enable rapid and high-resolution multidimensional coherent spectroscopy, Science 357, 1389 (2017).

[56] S. Sachdev, Quantum Phase Transitions, 2nd ed. (Cambridge University Press, New York, 2011).

[57] C. Chin, R. Grimm, P. Julienne, and E. Tiesinga, Feshbach resonances in ultracold gases, Rev. Mod. Phys. 82, 1225 (2010). 